\title{
Assessment of Heart Rate Variability in Young Patients with Primary Hypertension
}

\author{
Mansour M. Moustafa, and Mohamed Samy, Mohamed Omar \\ Cardiology Department, Faculty of Medicine, Al-Azhar University (Cairo) \\ Corresponding author: Mohamed Omar, Mob: 01149972692. Email: m.omercardio@ gmail.com
}

\begin{abstract}
Objective: this study aimed to determine the association between HRV and hypertension young adult patients, the effect of antihypertensive medications on HRV parameters was also studied. Subjects and Methods: 70 subjects under age of 40 years were classified into two groups: APatients' group, It included 50 subjected they were classified according to type of antihypertensive medications into 3 subgroups; 1): 20 patients on ACE-I, (2): 15 patients on BB, 3): 15 patients on CCB. B)- Control group included 20 healthy subjects. Pulse rate, BP, ECG and 24h Holter monitor were used to measure heart rate variability (HRV). Results: high statistical significant difference was found between patients and control according to SDNN rMSSD, pNN50 and LF/HF ratio ( $\mathrm{P}<$ 0.001). HRV parameters showed improvement in patient treated by BB and ACE-I, whereas no improvement was seen in patients treated by CCB. Conclusion: disturbed cardiac autonomic function was found in young hypertensive patients, treating such patients with either BB or ACE-I was associated with improvement of such autonomic imbalance.
\end{abstract}

Keywords: Primary hypertension, autonomic nervous system, ambulatory blood pressure, dipping, heart rate variability.

\section{INTRODUCTION}

The autonomic sensory system assumes a job in the pathogenesis of hypertension. Expanded thoughtful action or diminished parasympathetic movement adds to the advancement and support of hypertension ${ }^{(1)}$. Estimation of pulse inconstancy (HRV) in the recurrence area gives data on how the selfruling sensory system controls the cardiovascular framework ${ }^{(2)}$. In reality, the high-and low-recurrence parts of HRV separately mirror the action of the parasympathetic and thoughtful sensory system. The low-to high-recurrence proportion is a proportion of sympatho-vagal equalization (3)

In subjects in danger of hypertension and in hypertensive patients, the highrecurrence segment of HRV is commonly lessened. Decreased HRV predicts all-cause mortality and heart occasions ${ }^{(1)}$. Changes in thoughtful adjustment of the cardiovascular framework may consequently be a hazard factor for cardiovascular intricacies ${ }^{(4)}$, which may be reversible by circulatory strain bringing down treatment ${ }^{(\mathbf{1})}$. The present article gives an account of changes in HRV on youthful hypertensive grown-ups and impact of treatment by antihypertensive medications.

\section{Subjects and Methods}

This study included 70 young adult subjects $(<40$ years of age $)$ attending cardiology department of Al-Hussein University Hospital for assessment and followup of blood pressure. They were classified into two main groups:

The patients' group: constituted 50 primary hypertensive patients, they were further sub classified into 3 subgroups according to the treatment drug; (Subgroup1) included 20 patients on angiotensin-converting enzyme inhibitor (ACEI), (subgroup 2) included 15 patients on beta blockers (BB) and (subgroup3) included 15 patients on calcium channel blocker (CCB).

The control group: 20 normal subjects matched in age and sex with the patients group.

\section{Inclusion criteria:}

Patients diagnosed as HTN with BP $\geq 140 / 90$ or taking antihypertensive medication according to the latest ESC guidelines ${ }^{(5)}$.

\section{Exclusion criteria:}

Patients with secondary HTN patients, age > 40 years, Diabetic patients, chronic kidney disease, heart failure, atrial fibrillation, arrhythmia (atrial and ventricular), ischemic heart disease and obese patients.

\section{Methodology}

Informed consent was obtained from all participants then thorough history taking which focused on personal history (age, sex, 
smoking, occupation and special habit of medical importance), family history of hypertension, medical history of essential hypertension (HTN): Onset of essential hypertension and medical treatment (type of antihypertensive, dose, time of intake and compliance). Patient was considered HTN if his office systolic blood pressure was more than $139 \mathrm{mmHg}$ and diastolic blood pressure was more than $89 \mathrm{mmHg}{ }^{(5)}$. The study was approved by the Ethics Board of Al-Azhar University.

Clinical examination: pulse: rate, rhythm, equality, volume and special character, blood pressure and ambulatory blood pressure monitoring (ABPM). Blood pressure was measured according to the recommendation of European Society of Cardiology. Two blood pressure measurements spaced 1-2 min apart should be taken and additional measurements if the two are quite different ${ }^{(5)}$.

Laboratory assessment: fasting blood glucose level, serum creatinine, Lipid profile: In the form of serum LDL, HDL, cholesterol and triglycerides.

12-Lead Surface Electrocardiogram: Standard 12-lead electrocardiograms (ECG) studied for: Detection of the rate, rhythm and any recognized supra-ventricular or ventricular activity. QRS morphology, axis \& duration, left ventricular hypertrophy $(\mathrm{LVH})$ criteria by using Sokolow-Lyon criterion: SV1 + RV5 or $6>35 \mathrm{~mm}$

Conventional Echodoppler assessment.

24 hours Holter monitoring: the participants were subjected to 24 hours ambulatory 3channel Holter.

\section{Data analysis:}

Analysis of the whole period of 24 hours for detection of: Average, maximum and minimum heart rate. Detection of the sinus beats template and its number and identification of HRV with time domain in the form of SDNN >100msec. Thus, the observed cut-off values of 24-h measures of HRV e.g. SDNN $<50$ for highly depressed HRV, or SDNN $<100 \mathrm{~ms}$ for moderately depressed HRV are likely to be broadly applicable. PNN50\%=7.5. Frequency domain in the form of LF: ranging between 0.04 and $0.15 \mathrm{~Hz}, \mathrm{HF}$ : ranging from 0.15 to $0.4 \mathrm{~Hz}$ and $\mathrm{LF} / \mathrm{HF}$ ranging from (1.5-2) is considered as balanced ANS.

Ventricular arrhythmia: identifiable grading of ventricular arrhythmia risk according to the Lown's grade into: $0=$ no, ventricular premature beats (VPBs). $1 \leq 30 \mathrm{VPBs} /$ hour, 2 $\geq 30 \mathrm{VPBs} / \mathrm{h},-3=$ multiform VPBs, $4 \mathrm{a}=$ repetitive VPBs - couplets, $4 \mathrm{~b}=$ repetitive VBPs - runs of ventricular tachycardia, $5=$ early VPBs i.e. $\mathrm{R}$ on $\mathrm{T}^{\left({ }^{(6)}\right.}$.

\section{RESULTS}

The results of the present study were demonstrated in the following tables and figures.

Table 1: comparison between patients and control according to baseline characteristics

\begin{tabular}{|c|c|c|c|c|}
\hline Baseline characteristics & Patients $(n=50)$ & Control $(n=20)$ & $\mathbf{t} / \chi^{2} \#$ & p-value \\
\hline $\begin{array}{c}\text { Age }(\text { years }) \\
\text { Mean } \pm \text { SD } \\
\text { Range }\end{array}$ & $\begin{array}{c}27.30 \pm 6.24 \\
18-39 \\
\end{array}$ & $\begin{array}{c}30.30 \pm 4.89 \\
22-39 \\
\end{array}$ & 3.700 & 0.059 \\
\hline $\begin{array}{l}\text { Sex } \\
\quad \text { Female } \\
\text { Male } \\
\end{array}$ & $\begin{array}{l}25(50.0 \%) \\
25(50.0 \%)\end{array}$ & $\begin{array}{l}10(50.0 \%) \\
10(50.0 \%)\end{array}$ & 0.000\# & 1.000 \\
\hline $\begin{array}{l}\mathbf{B M I}\left[\mathbf{w t} /(\mathbf{h t})^{\wedge} \mathbf{2}\right] \\
\text { Mean } \pm \text { SD } \\
\text { Range }\end{array}$ & $\begin{array}{c}23.79 \pm 4.69 \\
17-34\end{array}$ & $\begin{array}{c}24.23 \pm 3.77 \\
19-31\end{array}$ & 0.139 & 0.711 \\
\hline $\begin{array}{l}\text { Smokers: no (\%) } \\
\text { No } \\
\text { Yes }\end{array}$ & $\begin{array}{l}43(86.0 \%) \\
7(14.0 \%)\end{array}$ & $\begin{array}{l}15(75.0 \%) \\
5(25.0 \%)\end{array}$ & $1.217 \#$ & 0.270 \\
\hline $\begin{array}{l}\text { EF\% } \\
\quad \text { Mean } \pm \text { SD } \\
\quad \text { Range } \\
\end{array}$ & $\begin{array}{c}64.82 \pm 10.92 \\
6-83 \\
\end{array}$ & $\begin{array}{c}62.60 \pm 5.85 \\
56-81 \\
\end{array}$ & 0.737 & 0.393 \\
\hline $\begin{array}{l}\text { Family History } \\
\text { No } \\
\text { Yes } \\
\end{array}$ & $\begin{array}{l}37(74.0 \%) \\
13(26.0 \%)\end{array}$ & $\begin{array}{l}15(75.0 \%) \\
5(25.0 \%)\end{array}$ & $0.007 \#$ & 0.931 \\
\hline
\end{tabular}


Assessment of Heart Rate Variability in Young Patients with Primary Hypertension

t-Independent Sample t-test; $\# \chi^{2}$ : Chi-square test, p-value $>0.05 \mathrm{NS}$

This table showed no statistically significant difference between patients and control according to baseline characteristics.

Table 2: comparison between patients and control according to laboratory work-up

\begin{tabular}{|l|c|c|c|c|}
\hline Lipid profile & Patients $(\mathbf{n}=\mathbf{5 0})$ & Control $(\mathbf{n}=\mathbf{2 0})$ & t-test & p-value \\
\hline $\begin{array}{l}\text { TG } \\
\text { Mean } \pm \text { SD }\end{array}$ & $109.18 \pm 3.11$ & $106.95 \pm 25.59$ & 0.085 & 0.772 \\
\hline $\begin{array}{l}\text { LDL } \\
\text { Mean } \pm \text { SD }\end{array}$ & $85.28 \pm 3.98$ & $78.95 \pm 14.00$ & 0.767 & 0.384 \\
\hline $\begin{array}{l}\text { HDL } \\
\quad \text { Mean } \pm \text { SD }\end{array}$ & $74.00 \pm 14.84$ & $73.50 \pm 8.57$ & 0.020 & 0.888 \\
\hline $\begin{array}{c}\text { Cholesterol } \\
\text { Mean } \pm \text { SD }\end{array}$ & $133.38 \pm 25.94$ & $157.20 \pm 22.07$ & 13.055 & $<0.001^{* * *}$ \\
\hline $\begin{array}{c}\text { Serum creatinine } \\
\text { Mean } \pm \text { SD }\end{array}$ & $0.83 \pm 0.1$ & $0.87 \pm 0.2$ & 0.468 & 0.496 \\
\hline $\begin{array}{c}\text { TSH } \\
\text { Mean } \pm S D\end{array}$ & $2.72 \pm 0.02$ & $2.84 \pm 0.98$ & 0.222 & 0.639 \\
\hline $\begin{array}{c}\text { Free T3 } \\
\text { Mean } \pm \text { SD }\end{array}$ & $3.51 \pm 0.87$ & $3.38 \pm 0.51$ & 0.396 & 0.531 \\
\hline $\begin{array}{c}\text { Free T4 } \\
\text { Mean } \pm \text { SD }\end{array}$ & $1.33 \pm 0.23$ & $1.40 \pm 0.22$ & 1.181 & 0.281 \\
\hline
\end{tabular}

t-Independent Sample t-test; p-value $>0.05 \mathrm{NS}$; **p-value $<0.001 \mathrm{HS}$

This table showed statistically significant difference between patients and control according to cholesterol.

Table 3: comparison between patients and control according to initial heart rate

\begin{tabular}{|l|c|c|c|c|}
\hline Initial Heart Rate & Patients $(\mathbf{n = 5 0})$ & Control $(\mathbf{n = 2 0})$ & t-test & p-value \\
\cline { 1 - 3 } Mean \pm SD & $78.46 \pm 7.15$ & $75.90 \pm 8.05$ & \multirow{2}{*}{1.705} & \multirow{2}{*}{0.196} \\
\hline Range & $65-95$ & $60-90$ & & \\
\hline
\end{tabular}

$t$-Independent Sample t-test; p-value $>0.05 \mathrm{NS}$

Table 4: comparison between initial treatment and after treatment according to blood pressure in patients group

\begin{tabular}{|l|c|c|c|c|c|}
\hline Blood Pressure & Initial $(\mathbf{n = 5 0})$ & $\begin{array}{c}\text { After } \\
(\mathbf{n = 5 0})\end{array}$ & $\begin{array}{c}\text { Mean } \\
\text { Diff. }\end{array}$ & t-test & p-value \\
\hline $\begin{array}{c}\text { Systolic blood pressure } \\
\text { Mean } \pm \text { SD }\end{array}$ & $154.40 \pm 11.94$ & $125.60 \pm 12.32$ & 28.80 & 11.038 & $<\mathbf{0 . 0 0 1 * *}$ \\
\hline $\begin{array}{c}\text { Diastolic blood pressure } \\
\text { Mean } \pm \text { SD }\end{array}$ & $97.20 \pm 8.15$ & $78.20 \pm 7.34$ & 19.00 & 11.665 & $<\mathbf{0 . 0 0 1 * *}$ \\
\hline
\end{tabular}

t-Paired Sample t-test; **p-value $<0.001 \mathrm{HS}$

This table showed statistically significant difference between initial and after treatment according to blood pressure.

Table 5: comparison between the patients and control according to initial treatment of heart rate variability

\begin{tabular}{|c|c|c|c|c|}
\hline $\begin{array}{l}\text { Initial treatment Heart rate } \\
\text { variability }\end{array}$ & Patients $(n=50)$ & Control $(n=20)$ & t-test & p-value \\
\hline $\begin{array}{r}\text { LF/HF Ratio } \\
\text { Mean } \pm \text { SD }\end{array}$ & $4.25 \pm 3.50$ & $1.90 \pm 0.28$ & 8.971 & $0.004 *$ \\
\hline $\begin{array}{l}\text { SDNN } \\
\quad \text { Mean } \pm \text { SD } \\
\text { Range }\end{array}$ & $\begin{array}{c}77.88 \pm 18.15 \\
40-120\end{array}$ & $\begin{array}{c}106.45 \pm 27.52 \\
1.9-136\end{array}$ & 25.964 & $<0.001 * *$ \\
\hline $\begin{array}{l}\text { RMSSD } \\
\text { Mean } \pm \text { SD } \\
\end{array}$ & $26.38 \pm 5.22$ & $42.95 \pm 11.98$ & 65.650 & $<0.001 * *$ \\
\hline $\begin{array}{l}\text { PNN50 } \\
\quad \text { Mean } \pm \text { SD }\end{array}$ & $3.99 \pm 1.63$ & $9.53 \pm 2.50$ & 119.869 & $<0.001 * *$ \\
\hline
\end{tabular}


This table showed statistically significant difference between patients and control according to initial treatment heart rate variability.

Table 6: comparison between the patients and control according to initial treatment of ambulatory blood pressure

\begin{tabular}{|l|c|c|c|c|}
\hline $\begin{array}{l}\text { Initial treatment of ambulatory } \\
\text { blood pressure }\end{array}$ & Patients $(\mathbf{n = 5 0 )}$ & Control (n=20) & t-test & p-value \\
\hline Day time & & & & \\
\hline $\begin{array}{l}\text { Systolic blood pressure } \\
\text { Mean } \pm \text { SD }\end{array}$ & $141.56 \pm 20.71$ & $125.30 \pm 9.27$ & 11.340 & $<\mathbf{0 . 0 0 1 * *}$ \\
\hline $\begin{array}{l}\text { Diastolic blood pressure } \\
\text { Mean } \pm \text { SD }\end{array}$ & $88.48 \pm 14.51$ & $79.95 \pm 8.02$ & 6.126 & $\mathbf{0 . 0 1 6 ^ { * }}$ \\
\hline $\begin{array}{l}\text { Mean arterial blood pressure } \\
\text { Mean } \pm \text { SD } \\
\text { Night time }\end{array}$ & $105.60 \pm 16.44$ & $94.75 \pm 7.94$ & 7.918 & $\mathbf{0 . 0 0 6 *}$ \\
\hline $\begin{array}{l}\text { Systolic blood pressure } \\
\text { Mean } \pm \text { SD }\end{array}$ & $135.90 \pm 21.98$ & $108.95 \pm 8.66$ & 28.119 & $<\mathbf{0 . 0 0 1 * *}$ \\
\hline $\begin{array}{l}\text { Diastolic blood pressure } \\
\text { Mean } \pm S D\end{array}$ & $82.94 \pm 14.56$ & $63.90 \pm 4.96$ & 32.456 & $<\mathbf{0 . 0 0 1 * *}$ \\
\hline $\begin{array}{l}\text { Mean arterial blood pressure } \\
\text { Mean } \pm \text { SD } \\
\text { Dipping\% }\end{array}$ & $98.90 \pm 16.99$ & $79.85 \pm 8.58$ & 22.687 & $<\mathbf{0 . 0 0 1 * *}$ \\
\hline $\begin{array}{l}\text { Dipping\% } \\
\text { Mean } \pm S D\end{array}$ & $6.65 \pm 2.54$ & $13.20 \pm 4.01$ & 67.148 & $<\mathbf{0 . 0 0 1 * *}$ \\
\hline Dipping category & $4(8.0 \%)$ & $18(90.0 \%)$ & 44.572 & $<\mathbf{0 . 0 0 1 * *}$ \\
\hline
\end{tabular}

t-Independent Sample t-test; *p-value $<0.05 \mathrm{~S} ; * *$ p-value $<0.001 \mathrm{HS}$

This table showed highly statistically significant difference between patients and control according to initial treatment of ambulatory blood pressure.

Table 4: comparison between initial treatment and after treatment according to ambulatory blood pressure in the patient's group

\begin{tabular}{|l|c|c|c|c|}
\hline Ambulatory blood pressure & $\begin{array}{c}\text { Initial treatment } \\
(\mathbf{n = 5 0 )}\end{array}$ & $\begin{array}{c}\text { After treatment } \\
(\mathbf{n = 5 0 )}\end{array}$ & $\mathbf{t}$-test & p-value \\
\hline Day time (Mean \pm SD) & & & & \\
\hline Systolic blood pressure & $141.56 \pm 20.71$ & $129.14 \pm 16.90$ & 6.546 & $<\mathbf{0 . 0 0 1} * *$ \\
\hline Diastolic blood pressure & $88.48 \pm 14.51$ & $85.90 \pm 14.04$ & 1.185 & 0.242 \\
\hline Mean arterial blood pressure & $105.60 \pm 16.44$ & $99.02 \pm 14.95$ & 3.714 & $<\mathbf{0 . 0 0 1} * *$ \\
\hline Night time (Mean \pm SD) & & & & \\
\hline Systolic blood pressure & $135.90 \pm 21.98$ & $121.02 \pm 15.29$ & 7.968 & $<\mathbf{0 . 0 0 1} * *$ \\
\hline Diastolic blood pressure & $82.94 \pm 14.56$ & $72.44 \pm 11.49$ & 6.158 & $<\mathbf{0 . 0 0 1} * *$ \\
\hline Mean arterial blood pressure & $98.90 \pm 16.99$ & $90.86 \pm 14.39$ & 3.502 & $<\mathbf{0 . 0 0 1} * *$ \\
\hline Dipping\% & $6.65 \pm 2.54$ & $10.24 \pm 4.51$ & -4.968 & $<\mathbf{0 . 0 0 1} * *$ \\
\hline Dipping category & $4(8.0 \%)$ & $31(62.0 \%)$ & $29.714 \#$ & $<\mathbf{0 . 0 0 1} * *$ \\
\hline
\end{tabular}

This table showed statistically significant difference between initial and after treatment according to ambulatory blood pressure. 
Table 5: comparison between sub-groups according to baseline characteristics in the patient's group

\begin{tabular}{|l|c|c|c|c|c|}
\hline $\begin{array}{c}\text { Baseline } \\
\text { characteristics }\end{array}$ & ACEI (n=20) & BB (n=15) & CCB (n=15) & F/ $\chi^{\mathbf{2} \#}$ & p-value \\
\hline Age (years) & $27.95 \pm 6.49$ & $27.93 \pm 6.37$ & $25.80 \pm 5.92$ & 0.609 & 0.548 \\
\hline Female & $10(50.0 \%)$ & $7(46.7 \%)$ & $8(53.3 \%)$ & $0.133 \#$ & 0.936 \\
Male & $10(50.0 \%)$ & $8(53.3 \%)$ & $7(46.7 \%)$ & & 0.140 \\
\hline BMI [wt/(ht)^2] & $25.35 \pm 5.66$ & $22.33 \pm 3.79$ & $23.15 \pm 3.60$ & 2.053 & 0.986 \\
\hline Smokers: No (\%) & $3(15.0 \%)$ & $2(13.3 \%)$ & $2(13.3 \%)$ & $0.028 \#$ & 0.639 \\
\hline EF\% & $63.00 \pm 14.77$ & $66.07 \pm 8.75$ & $66.00 \pm 6.07$ & 0.453 & 0.798 \\
\hline Family History & $6(30.0 \%)$ & $3(20.0 \%)$ & $4(26.7 \%)$ & $0.450 \#$ & 0 \\
\hline
\end{tabular}

F- One way Analysis of Variance; $\# \mathrm{x}^{2}$ : Chi-square test, p-value $>0.05 \mathrm{NS}$;

This table showed no statistically significant difference between patients sub-group according to baseline characteristics.

Table 6: comparison between sub-groups according to lipid profile in the patient's group

\begin{tabular}{|l|c|c|c|c|c|}
\hline Lipid profile & ACEI $(\mathbf{n = 2 0})$ & BB $(\mathbf{n = 1 5})$ & CCB $(\mathbf{n}=\mathbf{1 5})$ & ANOVA & p-value \\
\hline TG & $112.45 \pm 3.05$ & $96.73 \pm 9.81$ & $117.27 \pm 6.65$ & 2.021 & 0.144 \\
\hline LDL & $83.05 \pm 22.73$ & $86.07 \pm 28.80$ & $87.47 \pm 4.60$ & 0.091 & 0.914 \\
\hline HDL & $82.40 \pm 3.02$ & $74.00 \pm 13.62$ & $62.80 \pm 10.95$ & 10.321 & $<\mathbf{0 . 0 0 1 * *}$ \\
\hline Cholesterol & $137.40 \pm 33.52$ & $131.60 \pm 16.83$ & $129.80 \pm 22.50$ & 0.408 & 0.667 \\
\hline S. creatinine & $0.79 \pm 0.21$ & $0.90 \pm 0.19$ & $0.81 \pm 0.24$ & 1.318 & 0.277 \\
\hline TSH & $2.94 \pm 0.85$ & $2.36 \pm 0.11$ & $2.78 \pm 0.11$ & 1.413 & 0.254 \\
\hline Free T3 & $3.30 \pm 0.50$ & $3.30 \pm 0.44$ & $3.99 \pm 0.32$ & 3.726 & $\mathbf{0 . 0 3 1 ^ { * }}$ \\
\hline Free T4 & $1.34 \pm 0.26$ & $1.31 \pm 0.23$ & $1.35 \pm 0.20$ & 0.117 & 0.890 \\
\hline
\end{tabular}

F- One way Analysis of Variance; p-value $>0.05 \mathrm{NS}$; *p-value $<0.05 \mathrm{~S}$; **p-value <0.001 HS

This table showed statistically significant difference between patients sub-group according to HDL and free T3.

Table 7: comparison between sub-group according to heart rate and blood pressure in the patient's group

\begin{tabular}{|l|c|c|c|c|c|}
\hline & ACEI $(\mathbf{n = 2 0})$ & BB $(\mathbf{n = 1 5})$ & CCB $(\mathbf{n = 1 5})$ & ANOVA & p-value \\
\hline HR initial & $76.45 \pm 7.04$ & $81.47 \pm 7.72$ & $78.13 \pm 6.05$ & 2.241 & 0.118 \\
\hline HR after & $74.40 \pm 8.43$ & $69.47 \pm 6.16$ & $80.20 \pm 6.73$ & 8.088 & $<0.001 * *$ \\
\hline Initial SBP & $155.50 \pm 13.95$ & $155.00 \pm 10.52$ & $152.33 \pm 10.83$ & 0.320 & 0.728 \\
\hline Initial DBP & $98.25 \pm 7.12$ & $95.33 \pm 10.60$ & $97.67 \pm 6.78$ & 0.573 & 0.568 \\
\hline After SBP & $127.25 \pm 10.45$ & $122.00 \pm 10.49$ & $127.00 \pm 15.90$ & 0.914 & 0.408 \\
\hline After DBP & $78.50 \pm 7.63$ & $77.00 \pm 6.76$ & $79.00 \pm 7.84$ & 0.298 & 0.744 \\
\hline
\end{tabular}

ANOVA: One way Analysis of Variance; p-value >0.05 NS; **p-value <0.001 HS

This table showed statistically significant difference between patients sub-group according to heart rate after.

Table 8: comparison between sub-groups according to heart rate variability in patients group.

\begin{tabular}{|l|c|c|c|c|c|}
\hline $\begin{array}{l}\text { Heart rate } \\
\text { variability }\end{array}$ & ACEI $(\mathbf{n = 2 0})$ & BB $(\mathbf{n = 1 5})$ & CCB $(\mathbf{n = 1 5})$ & ANOVA & p-value \\
\hline Initial treatment & & & & & \\
\hline LF/HF Ratio & $4.84 \pm 5.50$ & $4.17 \pm 0.56$ & $3.56 \pm 0.77$ & 0.563 & 0.573 \\
\hline SDNN & $73.35 \pm 20.94$ & $77.60 \pm 17.62$ & $84.20 \pm 13.23$ & 1.570 & 0.219 \\
\hline RMSSD & $26.70 \pm 5.04$ & $25.80 \pm 5.36$ & $26.53 \pm 5.62$ & 0.132 & 0.877 \\
\hline PNN50 & $3.84 \pm 2.05$ & $3.85 \pm 1.14$ & $4.34 \pm 1.45$ & 0.484 & 0.619 \\
\hline After treatment & & & & & \\
\hline LF/HF Ratio & $1.55 \pm 0.45$ & $2.28 \pm 0.35$ & $3.69 \pm 0.30$ & 134.684 & $<\mathbf{0 . 0 0 1 * *}$ \\
\hline SDNN & $109.90 \pm 22.52$ & $110.73 \pm 17.83$ & $92.93 \pm 14.43$ & 4.365 & $\mathbf{0 . 0 1 8 *}$ \\
\hline RMSSD & $51.05 \pm 23.04$ & $31.73 \pm 5.89$ & $30.40 \pm 9.50$ & 9.543 & $<\mathbf{0 . 0 0 1 * *}$ \\
\hline
\end{tabular}


PNN50

$13.65 \pm 11.25$

$11.95 \pm 7.22$

$6.34 \pm 3.05$

3.453

0.040*

ANOVA: One way Analysis of Variance; $p>0.05 \mathrm{NS}$; * $<<0.05 \mathrm{~S} ; * * \mathrm{p}<0.001 \mathrm{HS}$

This table showed statistically significant difference between patients sub-group according to after treatment.

Table 9: comparison between initial treatment and after treatment according to heart rate variability in patient's sub-group

\begin{tabular}{|l|c|c|c|}
\hline Heart rate variability & ACEI $(\mathbf{n}=\mathbf{2 0})$ & $\mathbf{B B}(\mathbf{n = 1 5})$ & $\mathbf{C C B}(\mathbf{n}=\mathbf{1 5})$ \\
\hline LF/HF Ratio & & & \\
\hline Initial treatment & $4.84 \pm 5.50$ & $4.17 \pm 0.56$ & $3.56 \pm 0.77$ \\
\hline After treatment & $1.55 \pm 0.45$ & $2.28 \pm 0.35$ & $3.69 \pm 0.30$ \\
\hline$p$-value & $<\mathbf{0 . 0 0 1} * *$ & $<\mathbf{0 . 0 0 1} * *$ & 0.547 \\
\hline SDNN & & & \\
\hline Initial treatment & $73.35 \pm 20.94$ & $77.60 \pm 17.62$ & $84.20 \pm 13.23$ \\
\hline After treatment & $109.90 \pm 22.52$ & $110.73 \pm 17.83$ & $92.93 \pm 14.43$ \\
\hline p-value & $<\mathbf{0 . 0 0 1} 1^{* *}$ & $<\mathbf{0 . 0 0 1} * *$ & 0.095 \\
\hline RMSSD & & & $26.53 \pm 5.62$ \\
\hline Initial treatment & $26.70 \pm 5.04$ & $25.80 \pm 5.36$ & $30.40 \pm 9.50$ \\
\hline After treatment & $51.05 \pm 23.04$ & $31.73 \pm 5.89$ & 0.185 \\
\hline$p$-value & $<\mathbf{0 . 0 0 1} * *$ & $<0.001 * *$ & \\
\hline PNN50 & & & $3.34 \pm 1.45$ \\
\hline Initial treatment & $3.84 \pm 2.05$ & $3.85 \pm 1.14$ & $6.34 \pm 3.05$ \\
\hline After treatment & $13.65 \pm 11.25$ & $11.95 \pm 7.22$ & $\mathbf{0 . 0 2 9} *$ \\
\hline$p$-value & $<\mathbf{0 . 0 0 1} * *$ & $<0.001 * *$ & \\
\hline
\end{tabular}

Using: paired Sample t-test, p-value $>0.05 \mathrm{NS}$; *p-value $<0.05 \mathrm{~S}$; **p-value $<0.001 \mathrm{HS}$ This table shows statistically significant difference between initial treatment and after treatment according heart rate variability in ACEI and BB group, while CCB group significant in PNN50.

Table 10: comparison between patient's subgroups according to ambulatory BP

\begin{tabular}{|l|c|c|c|c|c|}
\hline Ambulatory BP & ACEI $(\mathbf{n}=\mathbf{2 0})$ & $\mathbf{B B}(\mathbf{n}=\mathbf{1 5})$ & $\mathbf{C C B}(\mathbf{n}=\mathbf{1 5})$ & \multicolumn{2}{c|}{ Significance test } \\
\hline Initial day time & Mean \pm SD & Mean \pm SD & Mean \pm SD & ANOVA & p-value \\
\hline SBP & $138.00 \pm 21.57$ & $147.73 \pm 22.19$ & $140.13 \pm 17.78$ & 0.997 & 0.377 \\
\hline DBP & $85.65 \pm 14.11$ & $92.67 \pm 15.73$ & $88.07 \pm 13.74$ & 1.012 & 0.371 \\
\hline MABP & $102.70 \pm 15.86$ & $110.47 \pm 17.02$ & $104.60 \pm 16.63$ & 0.996 & 0.377 \\
\hline Initial night time & & & & & \\
\hline SBP & $131.85 \pm 22.27$ & $140.73 \pm 26.26$ & $136.47 \pm 16.69$ & 0.699 & 0.502 \\
\hline DBP & $76.15 \pm 13.53$ & $90.40 \pm 13.69$ & $84.53 \pm 13.32$ & 4.912 & $\mathbf{0 . 0 1 2}$ \\
\hline MABP & $95.75 \pm 16.06$ & $104.13 \pm 18.28$ & $97.87 \pm 16.78$ & 1.087 & 0.345 \\
\hline Dipping\% & $7.01 \pm 3.35$ & $6.16 \pm 2.06$ & $6.67 \pm 1.62$ & 0.465 & 0.631 \\
\hline Dipping category & $3(15.0 \%)$ & $1(6.7 \%)$ & $0(0.0 \%)$ & 2.672 & 0.263 \\
\hline After day time & & & & & \\
\hline SBP & $123.85 \pm 16.21$ & $132.27 \pm 15.68$ & $133.07 \pm 18.18$ & 1.687 & 0.196 \\
\hline DBP & $87.15 \pm 10.25$ & $86.33 \pm 15.22$ & $83.80 \pm 17.57$ & 0.246 & 0.783 \\
\hline MABP & $95.85 \pm 9.38$ & $98.60 \pm 13.60$ & $103.67 \pm 20.99$ & 1.189 & 0.314 \\
\hline After night time & & & & & \\
\hline SBP & $118.65 \pm 16.07$ & $123.33 \pm 14.57$ & $121.87 \pm 15.54$ & 0.425 & 0.657 \\
\hline DBP & $68.45 \pm 6.98$ & $73.87 \pm 9.88$ & $76.33 \pm 16.04$ & 2.297 & 0.112 \\
\hline MABP & $86.00 \pm 8.45$ & $91.80 \pm 14.56$ & $96.40 \pm 18.67$ & 2.415 & 0.100 \\
\hline Dipping\% & $10.60 \pm 3.57$ & $11.65 \pm 5.30$ & $8.33 \pm 4.42$ & 2.951 & $\mathbf{0 . 0 4 6}$ \\
\hline Dipping category & $15(75.0 \%)$ & $12(80.0 \%)$ & $4(26.7 \%)$ & 11.446 & $\mathbf{0 . 0 0 3} *$ \\
\hline ANOVA On
\end{tabular}

ANOVA- One way Analysis of Variance; p-value $>0.05 \mathrm{NS}$; *p-value $<0.05 \mathrm{~S}$; 
This table shows statistically significant difference between patients sub-group according to DBP initial night time and dipping.

Table 11: comparison between initial treatment and after treatment according to dipping in patient's sub-groups

\begin{tabular}{|l|c|c|c|}
\hline \multicolumn{1}{|c|}{ Dipping \% } & ACEI $(\mathbf{n = 2 0})$ & BB $(\mathbf{n = 1 5})$ & CCB $(\mathbf{n = 1 5})$ \\
\hline Initial treatment & $7.01 \pm 3.35$ & $6.16 \pm 2.06$ & $6.67 \pm 1.62$ \\
\hline After treatment & $10.60 \pm 3.57$ & $11.65 \pm 5.30$ & $8.33 \pm 4.42$ \\
\hline$p$-value & $\mathbf{0 . 0 0 8}$ & $\mathbf{0 . 0 1 2} *$ & 0.183 \\
\hline
\end{tabular}

Using: paired Sample t-test, ${ }^{*}$ p-value $<0.05 \mathrm{~S}, * *$ p-value $<0.001 \mathrm{HS}$

This table shows statistically significant difference between initial treatment and after treatment according to dipping\% in patients sub-group ACEI and BB.

Table 12: correlation between initial dipping $\%$ with initial heart rate variability, using Pearson Correlation Coefficient in patients group.

\begin{tabular}{|l|c|c|}
\hline \multirow{2}{*}{ Initial Heart rate variability } & \multicolumn{2}{|c|}{ Initial Dipping\% } \\
\cline { 2 - 3 } & $\mathbf{r}$ & $\mathbf{p}$-value \\
\hline LF/HF Ratio & 0.429 & $\mathbf{0 . 0 0 2}^{*}$ \\
\hline SDNN & 0.317 & $\mathbf{0 . 0 2 5}^{*}$ \\
\hline RMSSD & 0.084 & 0.563 \\
\hline PNN50 & 0.405 & $\mathbf{0 . 0 0 3}$ \\
\hline
\end{tabular}

r-Pearson Correlation Coefficient, p-value $>0.05 \mathrm{NS}$; *p-value $<0.05 \mathrm{~S}$;

This table shows positive correlation and significant between initial dipping\% with $\mathrm{LF} / \mathrm{HF}$ ratio, SDNN and PNN50.

Table 13: correlation between after treatment dipping\% with after treatment heart rate variability, using Pearson Correlation Coefficient in patients group

\begin{tabular}{|l|c|c|}
\hline \multirow{2}{*}{ After Heart rate variability } & \multicolumn{2}{|c|}{ After Dipping\% } \\
\cline { 2 - 3 } & $\mathbf{r}$ & $\mathbf{p}$-value \\
\hline LF/HF Ratio & -0.188 & 0.191 \\
\hline SDNN & 0.712 & $<\mathbf{0 . 0 0 1 * *}$ \\
\hline RMSSD & 0.069 & 0.636 \\
\hline PNN50 & 0.441 & $<\mathbf{0 . 0 0 1 * *}$ \\
\hline
\end{tabular}

r-Pearson Correlation Coefficient, p-value $>0.05 \mathrm{NS} ; * *$ p-value $<0.001 \mathrm{HS}$

This table showed that there was positive correlation and significant between after dipping\% with

SDNN and PNN50.

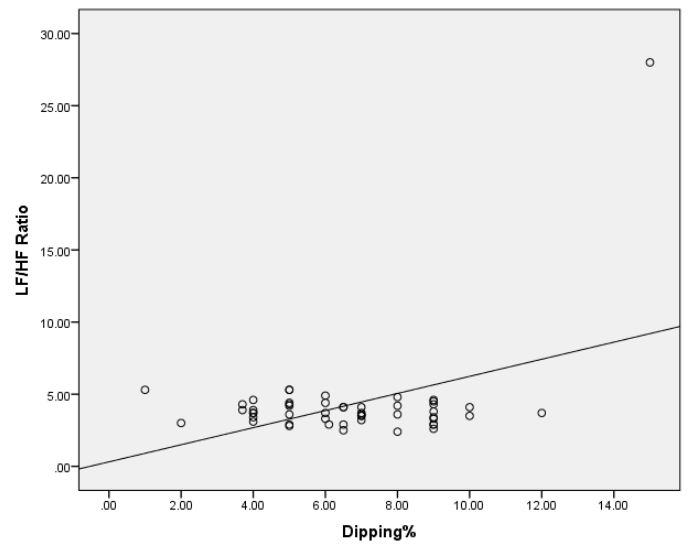

Fig. 1- Scatter plot between initial dipping\% and $\mathrm{LF} / \mathrm{HF}$ ratio in patients group

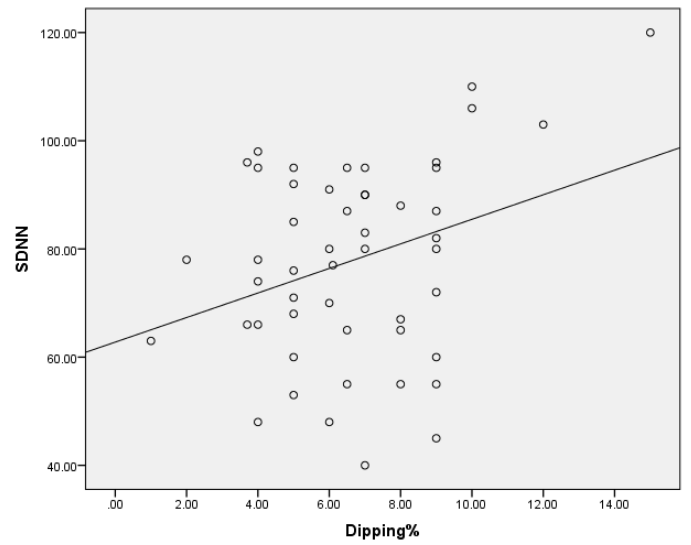

Fig.2- Scatter plot between initial dipping\% and SDNN in patients group 


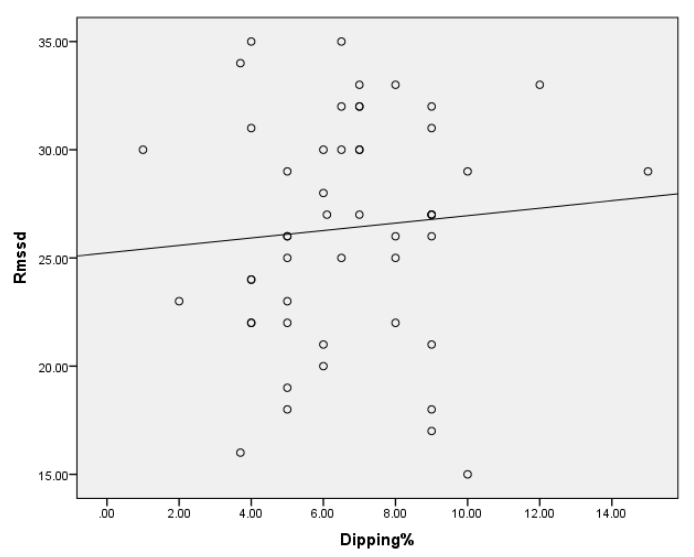

Fig. 3- Scatter plot between initial dipping\% and RMSSD in patients group

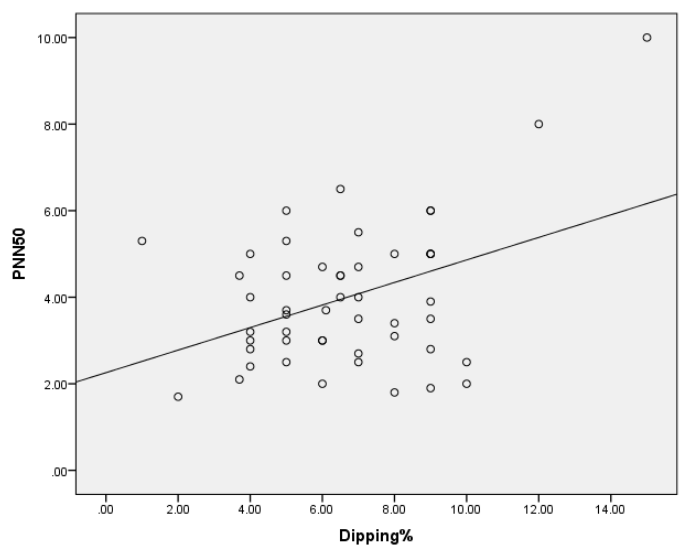

Fig. 4- Scatter plot between initial dipping\% and PNN50 in patients group

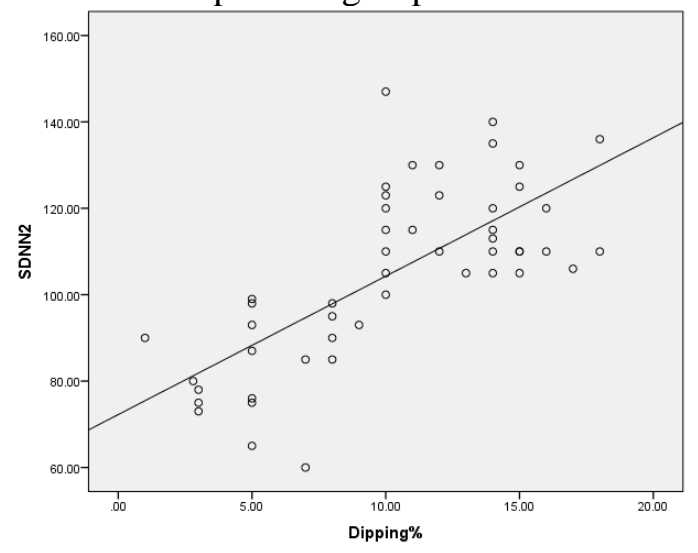

Fig. (5): Scatter plot between after dipping\% and SDNN in patients group

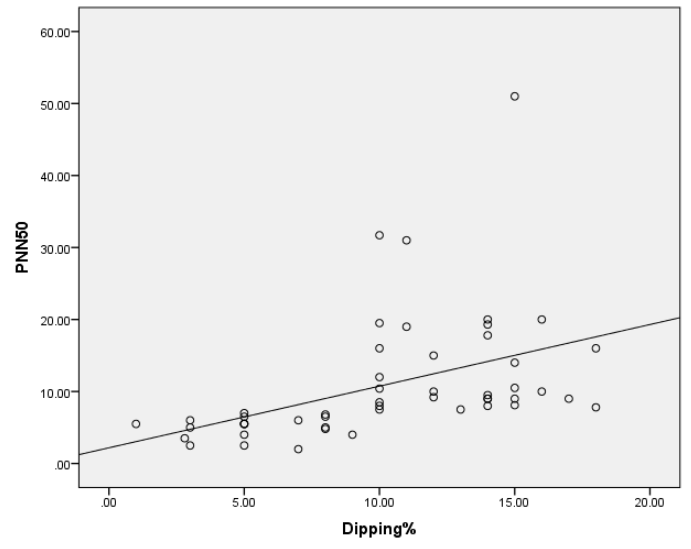

Fig. 6- Scatter plot between after dipping\% and PNN50 in patients group

\section{DISCUSSION}

Heart autonomic neuropathy is normal yet ignored complexity which adds to remaining danger for cardiovascular grimness and mortality (4). HRV estimated by straightforward $5 \mathrm{~min}$ recording gives solid status of cardiovascular autonomic parity ${ }^{(7)}$. Decreased HRV is seen in both HTN and T2DM exclusively and known to be an autonomous hazard factor for cardiovascular wellbeing ${ }^{(8)}$. In this examination we tried to decide the relationship among HRV and hypertension in youthful hypertensive grownup patients and to contemplate the impact of anti-hypertensive medications in such patients.

For this reason we picked 70 subjects less than 40 years old ordered into two gatherings: Patient's gathering; 50 oppressed with age extend from $18-39$ years with mean \pm SD of $27.3 \pm 6.24$. They were 25 guys and 25 females. They further sub-assembled by kind of treatment into subgroup 1- 20 patients on ACEI, subgroup 2- 15 on BB and subgroup 3- 15 on CCB. The control gathering: 20 typical subjects, sex and age coordinated were utilized as controls. Their age went from $22-39$ years and mean \pm SD of $30.3 \pm 4.89$ years.

Investigation showed no measurable contrast among patients and control bunches as respect age and sex $(\mathrm{P}>0.05)$. The investigation demonstrated no factually critical distinction among patients and control as indicated by gauge qualities (BMI, smoking, $\mathrm{EF} \%$, and in addition family ancestry of hypertension. The location rate of covered hypertension was higher in the more youthful patients than in the senior ${ }^{(9)}$. Also, there were numerous other impacting components, for 
example, smoking, liquor abuse, contraceptives, stationary way of life, weight, rest apnea and stress ${ }^{(10)}$. Thyroid parameters and diverse lipid parameters were insignificant $(\mathrm{P}>0.05)$ with the exception of in cholesterol as we found a factually noteworthy contrast among patients and control $(\mathrm{P}<0.001)$.

Like our outcomes, Longo et al. (11) revealed no noteworthy contrasts all in all clinical qualities of age, fasting plasma glucose (FPG), triglyceride (TG), add up to cholesterol (TC) and serum creatinine (s Cr) between the hypertensive patient's gathering and control gathering.

Our investigation demonstrated no measurably noteworthy distinction between patients subgroups $(1,2,3)$ in the benchmark attributes as respect serum creatinine and lipid profile $(\mathrm{P}>0.05)$, with the exception of HDL it indicated factually critical contrast $(\mathrm{P}<0.001)$. Thyroid hormones showed a factually irrelevant distinction $(\mathrm{P}>0.05)$ with the exception of $\mathrm{T} 3$ demonstrated a measurably huge contrast $(\mathrm{P}<0.05)$.

In an extensive all inclusive community companion in France, pulse was related with circulatory strain; hypertensive subjects had higher pulses than normotensive subjects, with the best increment found in those with moderate-serious hypertension ${ }^{(12)}$. Lift pulse was related with hoisted circulatory strain, expanded hazard for advancement of hypertension (and diabetes) and all-cause mortality ${ }^{(13)}$.Our investigation indicated measurably huge contrast between patient's subgroups $(1,2,3)$ as indicated by pulse after treatment $(\mathrm{P}<0.001)$.

Another examinations showed that more beta-blockers with vasodilating properties may bring down pulse and both fringe and focal circulatory strain, and expansion record ${ }^{(\mathbf{1 2})}$. In a hybrid investigation of 32 patients, atenolol was less powerful at bringing down aortic systolic circulatory strain and increase weight than ACEi, CCB and diuretics (14). An investigation of 393 patients with fundamental hypertension uncontrolled with $5 \mathrm{mg}$ amlodipine contrasted the mix of amlodipinevalsartan with amlodipine-atenolol. Following $24 \mathrm{wk}$, focal systolic circulatory strain was bring down in the amlodipine-valsartan $(\mathrm{P}=$ $0.013)^{(\mathbf{1 4})}$.

This indicates factually huge distinction between patien'ts subgroups $(1,2,3)$ as per HVR after treatment.Low pulse fluctuation was related with hypertension ${ }^{(\mathbf{1 5})}$. Low pulse changes are modifiable. A little report exhibited an expansion in pulse fluctuation with atenolol ${ }^{(16)}$. Changes in pulse identified with physical action and distinctive antihypertensive regimens alter circulatory strain and clinical results is obscure ${ }^{(17)}$.This examination indicated factually huge distinction between starting treatment and after treatment agreeing HRV in ACEI and BB subgroup ( $\mathrm{P}<0.001)$ as respect LF/HF Ratio, SDNN, RMSSD, PNN50, while in CCB subgroup was huge in PNN50 just $(\mathrm{P}<0.05)$.

Another investigation showed critical bends in HRV in patients with moderate hypertension when contrasted and that in ordinary control gathering, indicating significant changes in the autonomic capacity of hypertensive patients, reflected essentially by a highlighted decrease in SDNN, PNN50 and $\mathrm{LF}^{(18)}$.

Kudat et al. ${ }^{(19)}$ examined pulse changeability parameters among 31 hypertensive patients. They found that unequaled and recurrence space parameters aside from mean RR interim and the LF/HF proportion were fundamentally lower in HTN patients than in sound controls.Our examination found a factually huge distinction among patients and control as indicated by beginning treatment of pulse inconstancy ( $P$ $<0.001)$ and there was additionally a measurably noteworthy contrast among introductory and after treatment of the patients gathering $(\mathrm{P}<0.001)$.

This investigation showed that systolic and diastolic BP demonstrated a factually critical contrast among the patients and controls $(\mathrm{P}<0.001)$ and among starting and after treatment in the patients gathering ( $P$ $<0.001)$ with very factually critical contrast among patients and control as per introductory treatment of mobile pulse $(\mathrm{P}<0.001)$ in day time, evening, plunging $\%$ and plunging class, likewise among starting and after treatment of the patients gathering $(\mathrm{P}<0.001)$.In an investigation of 319 clinically normotensive volunteers, every one of them had 5 center estimations and 12-hour daytime wandering circulatory strain estimations, Selenta et al. ${ }^{(20)}$ found that $23 \%$ had veiled hypertension, characterized as a daytime circulatory strain $>135 / 85 \mathrm{~mm} \mathrm{Hg}$. Subjects with veiled hypertension would in general be male, past smokers, more established and they had 
expended more liquor. In opposing to our outcomes, Cuspidi et al. (21) did an examination on treated scoop and non-scoop basic hypertensive patients with various facility BP estimations. They didn't discover huge contrast in heart contribution between the two gatherings.

On correlation between patient's subgatherings $(1,2,3)$ as per walking circulatory strain in patients gathering, there was a measurably huge contrast between patient's sub-bunches as per DBP introductory evening time and plunging \%. This examination demonstrates measurably critical distinction between introductory treatment and after treatment as per dipping\% in patients; subbunches ACEI and BB not CCB $(\mathrm{P}<0.05)$.

We discovered positive relationship ( $r$ ) and centrality between introductory plunging $\%$ with LF/HF proportion, SDNN and PNN50 $(\mathrm{P}<0.01)$ and furthermore positive connection and noteworthiness between after dipping\% with SDNN and PNN50 (P <0.001). Thoughtful movement was reflected by time space pointers of SDNN, SDANN, SDNN INDEX, and RMSSD (22). Contrasting and normo-tensive, SDNN, SDANN, SNDD INDEX, and RMSSD were essentially diminished in mobile BP patients. Be that as it may, there was no measurably noteworthy contrast between the patients' sub-gatherings. They had autonomic sensory system brokenness, with expanded thoughtful movement and diminished vagus nerve action. A diminished HRV in AmHTN patients could be identified with an expansion in thoughtful anxious and renin-angiotensive framework action in hypertensive patients, and in addition a decline in the affectability of the baroreceptor ${ }^{(9)}$.Like our investigation, Matteucci et al. (23) assessed ABPM chronicles of normotensive subjects and hypertensive patients. They revealed that SBP and DBP were essentially higher in hypertensive and diabetic gatherings contrasted with control gathering.

Inverse to our outcomes, Nishioka et al. (24) broke down 24-h walking BP observing and detailed that beta-blockers were less viable in diminishing BP changeability and HRV than ACEIs and CCBs for patients with past stroke history.

Another report utilized power unearthly investigation of $\mathrm{HRV}$ recommended that a decrease in parasympathetic sensory system action may likewise add to the non-plunging BP design in fundamental hypertension (24). Hojo et al. (25) examined fourteen normotensive controls and 33 age-coordinated untreated hypertensive subjects to research the progressions in autonomic sensory system movement in fundamental hypertension. There were no critical contrasts in the 24-h mean LF/HF control proportion, LF power or HF control somewhere in the range of normotensive and hypertensive subjects. The 24-h LF/HF control proportion was essentially lower in non-scoops than in scoops. The mean daytime LF/HF control proportion was altogether lower in non-scoops than in scoops. The evening LF/HF control proportion was not essentially extraordinary between the two gatherings.

Ben Halima et al. ${ }^{(26)}$ examined 47 patients (30 scoops and 17 non-scoops). They inferred that non scoop had irregularity of circadian thoughtful vagal tone with higher nighttime thoughtful tone. The information may halfway clarify the higher frequency of cardiovascular occasions announced in nonscoop. Taking everything into account, this investigation recommended that following changes for confounders, exasperated cardiovascular autonomic capacity is related with expanded circulatory strain and revision of such unsettling influence could be accomplished utilizing BB and ACE-I.

\section{REFERENCES}

1. Osakwe CE, Jacobs L, Anisiuba BC et al. (2014): Newer Versus Older Antihypertensive Agents in African Hypertensive Patients Trial (NOAAH) Investigators. Heart rate variability on antihypertensive drugs in black patients living in sub-Saharan Africa. Blood Press, 23(3):174-80.

2. Ohta Y, Kawano Y, Hayashi S et al. (2016): Effects of cigarette smoking on ambulatory blood pressure, heart rate, and heart rate variability in treated hypertensive patients. Clin. Exp. Hypertens., 38(6): 5103.

3. de la Sierra A, Calhoun DA, Vinyoles $\mathbf{E}$ et al. 2014): Heart rate and heart rate variability in resistant versus controlled hypertension and in true versus white-coat resistance. J. Hum. Hypertens., 28(7):41620. 
4. Mori H, Saito I, Eguchi E et al. (2014): Heart rate variability and blood pressure among Japanese men and women: a community-based cross-sectional study. Hypertens. Res., 37(8): 779-84.

5. Mancia G and Grassi G (2013): Individualization of antihypertensive drug treatment. Diabetes Care, 36 (2): 301-6.

6. Adebayo RA, Ikwu AN, Balogun MO et al. (2015): Heart rate variability and arrhythmic patterns of 24-hour Holter electro-cardiography among Nigerians with cardiovascular diseases. Vasc. Health Risk Manag., 11: 353-9.

7. Pop-Busui R (2010): Cardiac autonomic neuropathy in diabetes: a clinical perspective. Diabetes Care, 33: 434-41.

8. Thayer JF, Hansen AL, Saus-Rose $\mathbf{E}$ et al. (2009): Heart rate variability, prefrontal neural function, and cognitive performance: the neuro-visceral integration perspective on self-regulation, adaptation, and health. Ann. Behav. Med., 37:141-53.

9. Eerola K, Rinne P, Penttinen AM et al. (2014): $\alpha$-MSH overexpression in the nucleus tractus solitarius decreases fat mass and elevates heart rate. J. Endocrinol., 222(1):123-36.

10. Yue WW, Yin J, Chen B et al. (2014): Analysis of heart rate variability in masked hypertension. Cell Biochem. Biophys.,70(1):201-5.

11. Longo $D$, Dorigatti $F$ and Palatini $P$ (2005): Masked hypertension in adults. Blood Pressure Monitoring, 10: 307-10.

12. Wolf $P$, Winhofer $Y$, Krššák $M$ et al. (2017): Heart lipids and hormones. Endocr. Connect., 6(4): 59-69.

13. Morcet JF, Safar M, Thomas $F$ et al. (1999): Associations between heart rate and other risk factors in a large French population. J. Hypertens., 17:1671-7.

14. Reule S and Drawz PE (2012): Heart rate and blood pressure: any possible implications for management of hypertension? Curr. Hypertens. Rep., 14(6):478-84

15. Morgan T, Lauri J, Bertram $\mathrm{D}$ et al. (2004): Effect of different antihypertensive drug classes on central aortic pressure. Am. J Hypertens., 17(2):118-23.

16. Brotman DJ, Bash LD, Qayyum $\mathrm{R}$ et al. (2010): Heart rate variability predicts ESRD and CKD-related hospitalization. J. Am. Soc. Nephrol., 21(9): 1560-70.
17. Lanza GA, Pitocco D, Navarese EP et al. (2007): Association between cardiac autonomic dysfunction and inflammation in type 1 diabetic patients: effect of betablockade. Eur. Heart J., 28(7): 814-20.

18. Menezes AS, Moreira HG and Daher MT (2004): Analysis of heart rate variability in hypertensive patients before and after treatment with inhibitors of angiotensin converting enzyme II (Portuguese). Arq. Bras. Cardiol.,83 (2):165- 73.

19. Kudat H, Akkaya V, Sozen AB et al. (2006): Heart rate variability in diabetes patients. J. Int. Med. Res., 34(3): 291-7.

20. Selenta C, Hogan $B$ and Linden W (2000): How often do office blood pressure measurements fail to identify true hypertension? An exploration of white-coat normo-tension. Arch. Fam. Med., 9: 53340.

21. Cuspidi C, Michev I, Meani S et al. (2003): Non-dipper treated hyper-tensive patients do not have increased cardiac structural alterations. Cardiovasc. Ultrasound, 14: 1-9.

22. Jihong $G$ and Ping $Z$ (2003): Dynamic Electrocardiography. Beijing: People's Med. Pub. House, pp: 196-224.

23. Matteucci E, Consani C, Masoni MC et al. (2012): Circadian blood pressure variability in type 1 diabetes subjects and their non-diabetic siblings. Diabetol. Metab. Syndr., 4: 1758-62.

24. Nishioka R, Kinoshita S, Shiibashi M et al. (2015): Evaluation of the differences in the effects of antihypertensive drugs on blood pressure variability by 24-Hour ambulatory blood pressure monitoring in chronic cerebro-vascular disease. J. Stroke Cerebrovasc. Dis., 24(8): 1848-54.

25. HojoY, Noma S, Ohki T et al. (1997): Autonomic nervous system activity in essential hypertension: compression between dippers and non-dippers. J. H. Hypertens., 11: 665-671.

26. Ben Halima A, Ben Miled $M$, Ben Halima M et al. (2010): The effects of dipper and non-dipper status on heart rate variability and heart rate turbulence in essential hypertension. Arch. Cardiovasc. Dis. Suppl., 2(1): 75-6. 